\title{
EVIDENCE FOR IMPURITY BANDS IN La-DOPED EUS*
}

\author{
R. R. HEIKES and C. W. CHEN \\ Westinghouse Research Laboratories \\ Pittsburgh, Penna. 15235
}

(Received 15 October 1964)

\begin{abstract}
In this note are presented experimental data on the electrical conductivity of Ladoped EuS. These and other data are discussed in terms of a model involving magnetization-dependent impurity banding.
\end{abstract}

\section{Introduction}

IN this note we present some recent experimental data on the electrical conductivity of La-doped EuS. In order to understand these data and other measurements on similar materials we present also a theoretical discussion of the the electrical properties of ferromagnetic semiconductors. It will be seen that for temperatures below the Curie temperature the existing ideas concerning impurity banding are valid if one properly takes into account the effects of the magnetization of the host lattice. In particular, the width of the impurity band varies rapidly with the degree of magnetic disorder and thus with magnetization. Above $T_{c}$, on the other hand, the electrons are considered to be in localized states and the conductivity process is of the sort proposed for $\mathrm{Li}$-doped $\mathrm{NiO}$ [1].

\section{Experimental}

Doped samples were prepared by mixing two stoichiometric sulfides, EuS and LaS, in appropriate proportions, followed by sintering in a closed Ta tube at $1700^{\circ} \mathrm{C}$. by means of rf induction heating. The 4-terminal method was employed to measure the resistance whenever possible. At resistances higher than $10^{5} \Omega$, the two terminal method was used. The resistivity data are reproducible within the experimental error and no hysteresis was observed during many cycles of heating and cooling.

In Figure 1 the (resistivity/temperature) of three doped compounds of doping concentrations 1,5 and 10 per cent are plotted against $1 / T$. The following should be noted:

(1) Above the maximum in the curve the data are well represented by a straight line of the form

$$
\rho / T=\rho_{0} e^{\Delta E / k T}
$$

The values of $\Delta E$ are shown on the figure.

(2) Although the appropriate magnetic measurements have not yet been made, it is reasonably certain that the temperature of the maximum in the curve is associated with $T_{c}$. Thus, the Curie temperature is seen to first increase and then decrease with increasing doping concentrations. An analogous behavior

\footnotetext{
* This research was supported by the Advanced Research Projects Agency, Director for Materials Sciences and was technically monitored by the Air Force Office of Scientific Research under Contract AF 49(638)-1245.
} 


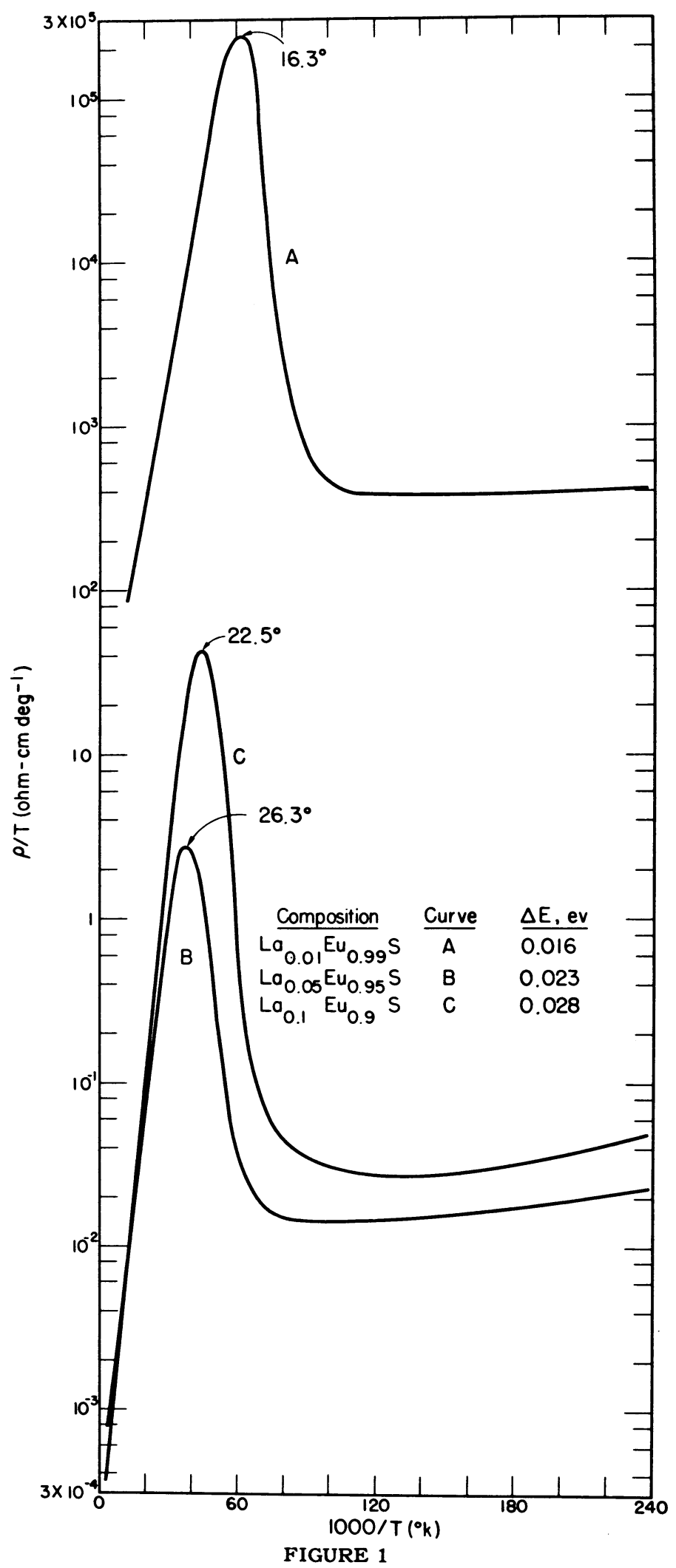

Observed curves of $\log \rho / T$ vs. $1 / T$ for three La-doped EuS compounds. 
has already been noted for the EuSe system [2]. This variation of $T_{c}$ is certainly indicative of a significant indirect coupling between the excess electrons and the f-shells.

(3) The resistivity anomaly is approximately three orders of magnitude in each case. Again (see (2)) it will be interesting to compare the shape of the anomaly with $M(T)$.

(4) It is to be noted that the ratio of the resistivity for the 1 per cent and the 5 per cent concentration is of the order of $10^{4}$ instead of 5 as might have been anticipated. As it would be difficult to attribute this discrepancy to a variation in mobility alone, we conclude that at 1 per cent concentration the carrier concentration is determined by accidental compensation due to impurities, the majority of carriers being immobilized by electrostatic interaction (we will call this region II). Such rapid variations of resistivity with concentration are quite common in impurity band conduction. (One could, of course, attribute this large ratio to poor sample preparation, that is, either poor control of the sulfur content or of the sintering conditions; however, similar results have been found in the EuSe system [2]. We are thus inclined to believe it to be real.) It is presumed that the 5 and 10 per cent concentrations are such that free quasi-metallic conduction occurs in the impurity band at low temperatures.

\section{Discussion}

As the concept of impurity banding is well known [3], it will not be necessary to go into detail here. Instead, only the novel features of the problem will be discussed. It has already been shown by Wolfram and Callaway [4] that the band width for the conduction electrons decreases rapidly (with a concomitant increase in the effective mass) as the magnetization of the host lattice decreases. This has two important consequences for ferromagnetic insulators such as the Eu chalcogenides. First, consider the radius of the wave function of the excess electron surrounding a donor impurity in the host lattice. Now, the radius is inversely proportional to the effective mass of the electron. Thus as the temperature is increased, with the consequent decrease in magnetization, the radius of the wave function will decrease. Second, as the band width continues to decrease with increasing temperature, we propose that finally in the neighborhood of the Curie temperature the band width becomes so small that the localized states are the stable ones; that is, the electrons are trapped on lattice sites.

An additional point to be considered with respect to the Eu compounds is the relatively small magnitude of the Weiss molecular field of the order of $5000 T_{c}$ Oe [5]. Thus in EuSe $\left(T_{c} \simeq 7^{\circ} \mathrm{K}\right)$ available magnetic fields can markedly affect the magnetization. In $\operatorname{EuS}\left(T_{c} \simeq 16^{\circ} \mathrm{K}\right)$ the effects will be less marked.

Using these ideas, let us now discuss the conductivity as a function of temperature and concentration. Consider first the concentration region of extreme dilution. (We will refer to this as region I.) By this we mean that the excess electron wave functions on neighboring donors have negligible overlap. Under these conditions conduction below $T_{c}$ can take place by two mechan isms: (1) the thermal excitation of the excess electrons into the conduction band, (2) if there is compensation, one can have impurity hopping. In general one should expect to observe both these mechanis ms in their appropriate temperature ranges as one does in the diamond-type semiconductors. It should be remarked that as the temperature increases, the activation energy for impurity hopping increases due to the decrease in the radius of the excess electron wave function. Above $T_{c}$ we assert that the mechanism changes to a thermally activated diffusion from site to site. None of our samples were in Region I at $T \ll T_{c}$.

We consider two additional concentration regions: (1) Region II where the overlap is sufficiently large so that if there is compensation, conduction can take place in the impurity band without activation energy. (The number of carriers present is then determined by the degree of compensation.) (2) Region III where the overlap is so large that the excess electrons essentially form a degenerate gas.

In Region II the correlation energy is still a dominant term so that compensation is necessary in order that one may have significant electrical conductivity. Now, at the lowest temperature one can have an unactivated conductivity; however, as the temperature is increased, the radius of the excess electron wave function decreases and effectively one returns to the case of Region I. As the temperature continues to increase, the impurity hopping activation energy increases until one reaches the Curie temperature. At or near this temperature the localized states become the stable ones. Above this temperature the 
activation energy is composed of two terms: (1) ionization energy from an impurity [6], (2) activation energy for hopping from lattice site to lattice site. This latter term is, of course, not to be confused with the activation energy associated with impurity conduction involving hopping from impurity to impurity.

Now, as the concentration increases and the correlation energy becomes less important, we come into the "metallic" Region III. From the discussion of the preceding paragraph, the temperature dependence of this case may easily be described.

As we mentioned, we believe the 1 per cent sample we measured is in Region II at low temperature, the others in III. All experience the breakup of the impurity band and the transition to Region I as they approach $T_{c}$. In the high temperature region conduction is of the typical "hopping" form in all cases.

Finally, let us consider the effect of a magnetic field on the electrical properties. We have already remarked that the magnetic field can strongly influence the magnetization in the Eu compounds. Thus, the effect of an external magnetic field can be considered as equivalent to a decrease in the temperature of the compound. Thus, it is clear that the application of a magnetic field at the Curie temperature might cause order of magnitude changes in the electrical conductivity. Furthermore, since the magnetic antisotropy of the Eu compounds is small, the electrical anomaly should be essentially independent of the relative orientation of the electric current and the magnetic field. No measurements in a magnetic field have been reported on the EuS system; however, Suits [7] has reported on such measurements in the EuSe system. His results appear to be in accord with this discussion.

The authors are indebted to Dr. F. L. Carter for the preparation of the samples.

\section{References}

1. R. R. HeIKeS, A. A. MARAdUdin and R. C. MILler, Ann. Phys. 8, 733 (1963).

2. T. R. McGUIRE, private communication.

3. T. KASUYA, J. Phys. Soc. Japan, 13, 1096 (1958); T. KASUYA and S. KOIDE, Ibid. 13, 1287 (1958).

4. T. WOLFRAM and J. CALlaWAY, Phys. Rev. 127, 1605 (1962).

5. S. VAN HOUTEN, Phillips Res. Rep. 17, 451 (1962).

6. At high impurity concentrations this term needs to be modified: cf. R. MILLER, R. HEIKES and R. MAZELSKY, J. Appl. Phys. 32, 2202 (1961).

7. J. C. SUITS, Bul1. Amer. Phys. Soc. Ser. II, 8, No. 4, 381 (1963). 